\title{
Quality of User Experience in 5G-VANET
}

\author{
Madiha Shahzad, Josephina Antoniou \\ School of Sciences \\ University of Central Lancashire \\ Larnaca, Cyprus \\ \{mshahzad1,jantoniou\}@uclan.ac.uk
}

\begin{abstract}
The coalescence of 5G networks and vehicular ad-hoc networks (VANETs) will result in intelligent transportation and safety services and in-vehicle entertainment services. As a result, the plethora of connected devices (cars, mobile phones and other communication devices/sensors) will benefit from off-loading of network data on unlicensed bands to support network load balancing, providing guaranteed bit rate services and a reduction in control signaling, hence improving the overall user experience. In this paper we briefly discuss the enabling technologies, various communication scenarios within the 5G-VANET and the crucial user experience perspective. It should be noted that service acceptance depends heavily on user opinion formulated as per their experience. We further address the multi-layer Quality of Experience (QoE) assessment model and propose the way forward to enhance user experience within 5G-VANET. Since it is a work in progress, we discuss the importance of how and where the network performance measurements should be made and their effect on the overall user experience with future contributions in form of network simulations.
\end{abstract}

Keywords-5G networks, VANET, User experience, V2X, cooperative computing

\section{INTRODUCTION}

The 5G cellular networks can provide high-capacity, low-latency communication for vehicles in highly mobile environments and has potential to meet the requirements laid out for the intelligent transport system (ITS) [1]. In such an environment, the vehicles themselves have a dedicated communication unit and each passenger can have at least one mobile device. The modern car is a complex sensor platform, which obtain information from the environment, including other vehicles and process, manage and transfer that information to not only drivers but also to the infrastructure [2]. This information exchange assist in safe navigation, pollution control and traffic management [2] among other usage. Couple this scenario with intelligent road infrastructure (traffic signals with sensors and communication unit) and various sensors within the environment (intelligent cities), the number of devices will be huge (Internet of Things [3]). The swelling in-vehicle data and its management will not be viable for the future 5G networks firstly because of the limited spectrum resources, even though it operates on three different spectrum bands. Secondly, consider a dense urban scenario where all the vehicles build connections with an eNodeB (eNB), traffic congestion and packet losses will occur more frequently. Furthermore, bring vehicle mobility with frequent handovers as the by-product in this equation and the network will have to deal with additional signaling overhead exhausting the precious channel resources [4].

To overcome the above-mentioned problems brought by using $5 \mathrm{G}$ network to carry vehicular data, one vehicle can be selected as a mobile gateway for a group of vehicles and can be connected with the eNB, thus the other vehicles in this group send and receive data through this gateway using short range radio technologies specifically for inter-vehicle communications; a merger of 5G networks and VANETs. Extensive amount of research has been conducted in both these domains. One promising approach is to utilize the IEEE 802.11p-based Dedicated Short-Range Communication (DSRC) which uses the spectrum of $75 \mathrm{MHz}$ at $5.9 \mathrm{GHz}$, communication range around 300 meters and data rate ranging from 6 to 27 Mbps [5]. The research community has proposed numerous design and communication solutions and they will be discussed in Section 2. However, it should be noted here that this paper does not aim to propose another model for 5G and VANET integration, rather it builds upon the existing 5G-VANET architectures to analyze and assess the support of QoE management. We are interested in the overall user experience and how it can be enhanced in such a network.

It is pertinent to mention the paradigm of mobile edge computing (MEC) within a $5 \mathrm{G}$ network along with software defined networks (SDN) and network virtualization. Significant amount of computing power will be distributed near the vehicles in the network with control and data plane abstractions along with a generalization of network hardware. With MEC majority of data will be processed and stored at the edge, which can reduce latency and provide better quality of service for connected vehicles [6] and all other users. Specially in dense urban scenarios, with small 5G cells and localized data centers, faster and more responsive service can be offered. Consequently, the improved real-time scheduling over the caching and transmission will boost the overall user experience. Another relevant concept is data offloading; whereby cellular traffic is diverted to unlicensed Wi-Fi spectrum or other network technologies, which helps to reduce the load on the existing cellular bands and result in better resource and spectrum utilization [7].

The distinguishing feature of this paper is the exploration of 5G-VANET with respect to overall user experience. We identify a generic model and build upon it with QoS parameters. Section 2 will cover the concepts and some relevant research on 5G networks in general and 5G-VANETs in particular. Section 3 discusses the quality of experience 
requirements posed within a 5G-VANET and a generic model which identifies the system layers where network performance should be measured in order to quantify user experience. Section 4 discusses the layered model in further detail with the relevant system influence factors and a QoE model. With the flexibility offered by 5G networks in terms of SDN and NVF, our aim is to analyze where, when and what quality of service (QoS) parameters should be measured, how it effects the overall system performance and if some are more important than others. Since it is a work in progress, simulation modelling is currently underway. Conclusions are drawn in Section 5 along with the challenges and future work.

\section{RELATED WORK}

This section documents the enabling technologies offered by $5 \mathrm{G}$ networks and the relevant research conducted within the domains of 5G-VANETs.

\section{A. $5 G$ Building Blocks}

Gradual roll-out of mobile 5G (in its various forms and flavors) has started in various cities around the world. Along with it, the standardization efforts for key networks enablers is taking final shape or have recently been finalized. As previously discussed, this research exploits these core building blocks offered by 5G network as they enable the future 5G-VANET and ITS. In this section we will briefly discuss these concepts and techniques to lay a foundation for our work.

1) Mobile Edge Computing/Fog Computing

MEC provides a platform for bringing services to the most suitable network location such as the mobile vehicles on roads in a multi-vendor environment. The mobile edge platform is responsible for the discovery, access and advertisement of MEC services [8]. With sufficient computing resources, MEC outperforms in-vehicle computing capabilities in the execution of computation-intensive tasks and in delivering lower execution latency. Also, because the distance between MEC resources and vehicles is shorter than the distance between vehicles and servers, MEC significantly outperforms cloud servers in terms of transmission latency.

The idea of vehicular fog computing is proposed in [9] which employs under-used vehicles as the infrastructure for task computation and communication. A real-time traffic management solution in a MEC-enabled vehicular network is proposed in [10] with the aim to minimize the average response time of the reported events by the vehicles. Some initial studies have also been carried out to integrate either of these technologies into vehicular communication networks [4], [10]- [12].

\section{2) Network Slicing}

The key challenge in $5 \mathrm{G}$ is the management of all the available heterogeneous access networks. Network slicing aims to ease management by logically separating the networks. In particular, the control plane, which is available to the network devices, is split into multiple control planes for specifying the forwarding rules for the designated data plane. Technologies such as SDN and NFV can be used for network slicing. Transport safety applications can be specified as a network slice that requires lower latency and highly reliable periodic message transmission. Another logical network slice can be designated for infotainment applications to satisfy their QoS requirements and to enhance user experience with high bandwidth demanding rich content distribution. [13].

\section{3) Software Defined Network}

SDN is a layered network structure, where the control layer provides efficient centralized management over the underlying infrastructure through software modules. Ku et. al. proposed a SDN-based VANET architecture in [14] and highlighted that SDN-based routing is advantageous in packet delivering ratio compared with traditional routing protocols. A VANET architecture supporting SDN and fog computing technologies was proposed in [15]. Resource management and fog coordination for both safety and non-safety services were also analyzed in this architecture. Merits of this architecture include reducing latency and improving resource utilization rate. A fog-enabled real-time traffic management system is proposed in [10] and [16]. A hierarchical SDN-based vehicular architecture is presented in [17], solving the problem of connectivity loss between forwarding switches and the controller. Simulation results showed that the approach performed better than traditional solutions without SDN controllers.

\section{4) Network Functions Virtualization}

NFV consists of the network functions that form a slice or a service to be implemented in a virtualized manner as Virtual Network Function (VNF) and considered a software application[18]. ETSI NFV is actively working on the definition of Network Service Template (NST) that could allow the instantiation of services, understanding them as a composition of several linked VNFs, including some information regarding the (virtual) links connecting them in the forwarding graph. NFV and SDN allow different tenants to share the same general purpose hardware and in combination these technologies can allow to build fully decoupled end-to-end networks on top of a common, shared infrastructure. The intelligent on-board system (IOS) in vehicles can be treated as a VNF resulting in open IOS design and efficient upgrades as proposed in [35].

\section{B. $5 G$ Vehicular Ad hoc NETworks}

VANETs are a particular class of Mobile Ad hoc NETworks (MANETs), characterized by high (variable) vehicle speed, hostile propagation environment, and quickly changing network topology [19]. The significant penetration of mobile devices means that each vehicle can be treated as a small cluster of mobile devices (UEs, including cars and other sensors) specially when considering public-mode of transportation. Furthermore, the support of self-driven cars, or remotely 
controlled vehicles will pose additional service requirements of low latency and high reliability. Extensive research has been conducted on media access control (MAC) and physical layer issues, routing algorithms, clustering algorithms, mobility management, data management and novel applications and services for VANETs. However, our research does not intend to propose a new model or protocol rather its focus on user experience in such a domain. In order to lay the foundation for this work, we will briefly discuss the requirements, communication paradigms and some relevant 5G-VANET systems.

1) Requirements

Several vehicular communication system requirements are pertinent to both transportation safety and non-safety applications, as they directly have a impact on user experience. These requirements include 1) minimizing communication load, 2) frequent seamless handover, 3) congestion control mechanisms, 4) fairness in accessing resources, 5) reliability and 6) support for diverse applications [7].

2) Communication Model - Vehicle-to-Everything (V2X)

3GPP identifies four types of V2X communication modes: vehicle-to-vehicle (V2V), vehicle-to-pedestrian (V2P), vehicleto-infrastructure (V2I) and vehicle-to-network (V2N) [13]. V2V and V2P modes cover direct communication between vehicle user equipment (UEs) and between vehicles and road users without the involvement of any network infrastructure. In a dense urban scenario, the number of pedestrians, bikers or motorcyclists will be high as compare to a rural setting.

V2I refers to communication between vehicles and the road side infrastructure, for example, a roadside unit (RSU) implemented in an eNodeB or a standalone communication unit. V2N is the communication between vehicular UE and a server supporting V2N applications. The servers can be localized and placed at the edge exploiting the benefits offered by MEC.

$\mathrm{V} 2 \mathrm{~V}$ and V2I communication patterns require radio access technology to provide ubiquitous network coverage and efficient spectrum, utilization. Furthermore, the communication can be in-vehicle, for example, with on-board unit, Global Positioning System (GPS) unit, in-car entertainment system, etc or with devices (or infrastructure) external to the vehicle.

\section{3) 5G-VANET Systems}

Numerous 5G-VANET systems have been proposed in the literature. As previously discussed, network slicing logically isolates network functions and resources and couples them together to best suit the service requirement, hence a slice potentially spans all 5G network domain across the core network $(\mathrm{CN})$ and radio access network (RAN) segments [13] and a VANET. The segregation of control plane and data plane should be reiterated here.

SDN is introduced into the 5G-VANET system [14] in order to enable the coordination and information sharing between base stations (BSs) to guarantee adaptive and efficient clustering. The SDN-enabled 5G-VANET system logically includes application plane, control plane, and data plane. Aujla et. al. in [7] proposes a SDN-based controller for data offloading in VANET.

Ning et.al. proposes a non-orthogonal multiple access (NOMA) technique for MEC-enabled vehicular network in [21]. A $\mathrm{V} 2 \mathrm{~V}$ enabled predictive offloading scheme is devised where various traffic/environment events can be recorded by the driver or passengers and offloaded within the macro-cell. Bouk et. al. in [22] and Amadeo, et. al. in [23], proposes architectural modifications to use Named Data Networking (NDN) in VANETs, which is a realization of Information-centric Networking. A NDN uses human-readable hierarchical names to tag, discover and deliver data/content.

The data generated, exchanged and managed within a VANET depends on the type of application; notably, transportation safety applications and infotainment applications. With 5G support, future networks will be able to accommodate autonomous/remote driving applications. The properties, behaviour and requirements of these applications are different and depends on the type of content and hence the quality of service (QoS) requirements will vary.

In the domain of VANETs, Garai and Boudriga coupled QoS levels with vehicle clusters in a VANET by measuring delay, throughput and packet loss for each cluster and periodically update them [24].

\section{QUALITY OF EXPERIENCE}

Quality of Experience (QoE) of a user is an important metric to measure how well a communication system performs and its overall acceptability and usability can be judged. It includes the complete end-to-end system; the user, the end-device, underlying network infrastructure(s), services and application/content.

ITU has proposed two methods to measure user experience; 1) subjective QoE assessment, typically based on Mean Opinion Score (MOS) of a service according to user perception and 2) objective QoE assessment, typically involving Quality of Service (QoS) parameters like latency, traffic volume density, reliability and cost etc [25]. A number of survey papers have been published in the past 2-4 years giving various overviews of QoE management-related studies. An in-depth overview and comparison of previous studies is given by Barakovic and Skorin-Kapov [26], with focus in particular on QoE modeling, monitoring, and control in wireless networks.

5G communication networks offers efficient use of radio spectrum, wider coverage and much faster data rates with stable connections. Positive user experience is crucial for technology acceptance and consumer willingness to pay higher service 
charges. Pierucci in [27] highlighted 5G features such as, high data rates, heterogeneous network architectures, ultra-low latency, device to device (D2D) communication, smart devices, and flexible spectrum management as challenges to QoE. Andriyanto and Suryanegara argues that QoE measurements in $5 \mathrm{G}$ communication network should be according to the three basic scenarios proposed by ITU i.e. 1) Enhanced Mobile Broadband, 2) Ultra-reliable and low latency communications, and 3) massive machine type communications [28]. Liotou et. al recommends a shift from system-centric architecture to usercentric architecture for $5 \mathrm{G}$ ecosystem and identify some QoE requirements. They identified SDN as the key technology for QoE management and provisioning functions and outlined consistency, transparency, user personalization and service differentiation and resource and energy-efficient QoE-awareness as the key requirements [29].

QoE requirements and management in a 5G-VANET is further complicated by the mobility, volatility and scalability [28]. In order to quantify QoE and translate it into user experience, QoE management has been approached from multiple complimentary perspectives. In [30], Skorin-Kapov et. al. lists them as; QoE-driven application management, QoE-driven network management, QoE-driven user monitoring and cross-layer QoE management. Most research conducted in this area supports multi-layer or cross-layer QoE management [30]. Inspired by the existing proposals, Figure 1 illustrates a generic QoE model which leverages the 5G features and support multiple vendors/stake-holders in the business model. Multi-layer (including cross-layer) QoE models have been proposed in the literature previously and usually comprises of QoE modelling, monitoring and management components. An extensive survey is conducted by Skorin-Kapov in [26, 30].

It should be highlighted that in 5G-VANETs in addition to the typical mobile network operators (MNOs), application providers and content providers, car manufacturers and local municipalities or governments will also be involved as the owner of road side units (RSUs) and intelligent traffic infrastructure. Hence we have included a business model in Figure 1, which has policies or agreements with respect to all the stakeholders involved. In case of any conflicts, specially in terms of network resource usage for certain applications, usage during peak hours, or special events, etc, can be resolved.

From VANET perspective, the protocols and architectures proposed in the literature follows the traditional layered communication model of service plane, transport plane and delivery plane. Often the solutions are scenario or application specific. When we integrate VANET with $5 \mathrm{G}$ networks, the core concepts or SDN, NFV and MEC with transform the layered architecture, as discussed in the following section.

\section{QOE ASSESSMENT MODEL}

Identification of relevant QoE parameters and the degree of their impact is established and defined within the QoE model which then help shape the QoE monitoring and management approaches. Generic relationship between QoE and QoS has been intensively discussed in literature [30], but mainly with a focus on single QoS parameters. However, the organic relationship between them, and how to best model it is not yet clear.

\section{A. 5G-VANET QoE Measurement}

In this section we will document the system and network parameters that will influence the overall user experience specifically for 5G-VANETs. As previously discussed, QoE is measured both subjectively and objectively. Figure 2 has a list of the system influence factors which are used for objective QoE assessment. Please note that this is not an extensive list and some parameters will be measured at multiple network devices. Subjective QoE assessment will be conducted in the later part of this research and at present is not a part of this paper.

The main performance metrics that needs to be collected are the radio performance measurements and application performance measurements coupled with user preferences and pricing models.

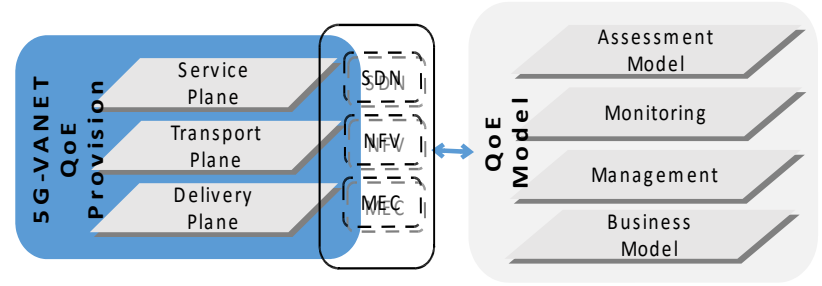




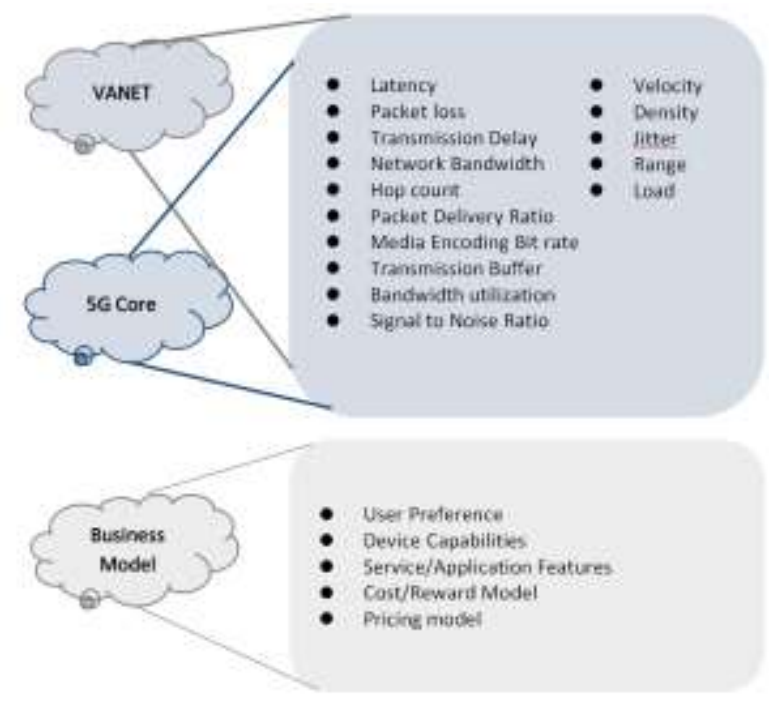

Figure 2: QoE System Influence Factors

\section{B. 5G-VANET QoE Model}

In order to support future networks, network operators are increasingly turning to SDN-based solutions facilitating centralized control of a dynamically programmable forwarding network. Such a network has a controlling entity called an SDN controller (SDNC). It has two interfaces 1) southbound application programming interface (API), which is used to transfer data to network switches or router and 2) the northbound API, which is used to communicate with the applications and business logic. By shifting intelligence to a centralized unit, service chains of virtualized functions can be controlled by SDNC and dynamically orchestrated in real-time. Additionally, QoE-driven control decisions can be made based on a global view of the underlying network state. Monitoring data needs to be fed to a control plane that is capable of driving QoE control. Furthermore, with standardized northbound APIs, mechanisms are provided for applications to provide requirements to the controller (driven by QoS-to-QoE mapping models), which can in turn invoke traffic management mechanisms to meet differentiated service requirements [30]. Figure 3 portrays a QoE model in the context of SDN and VNF.

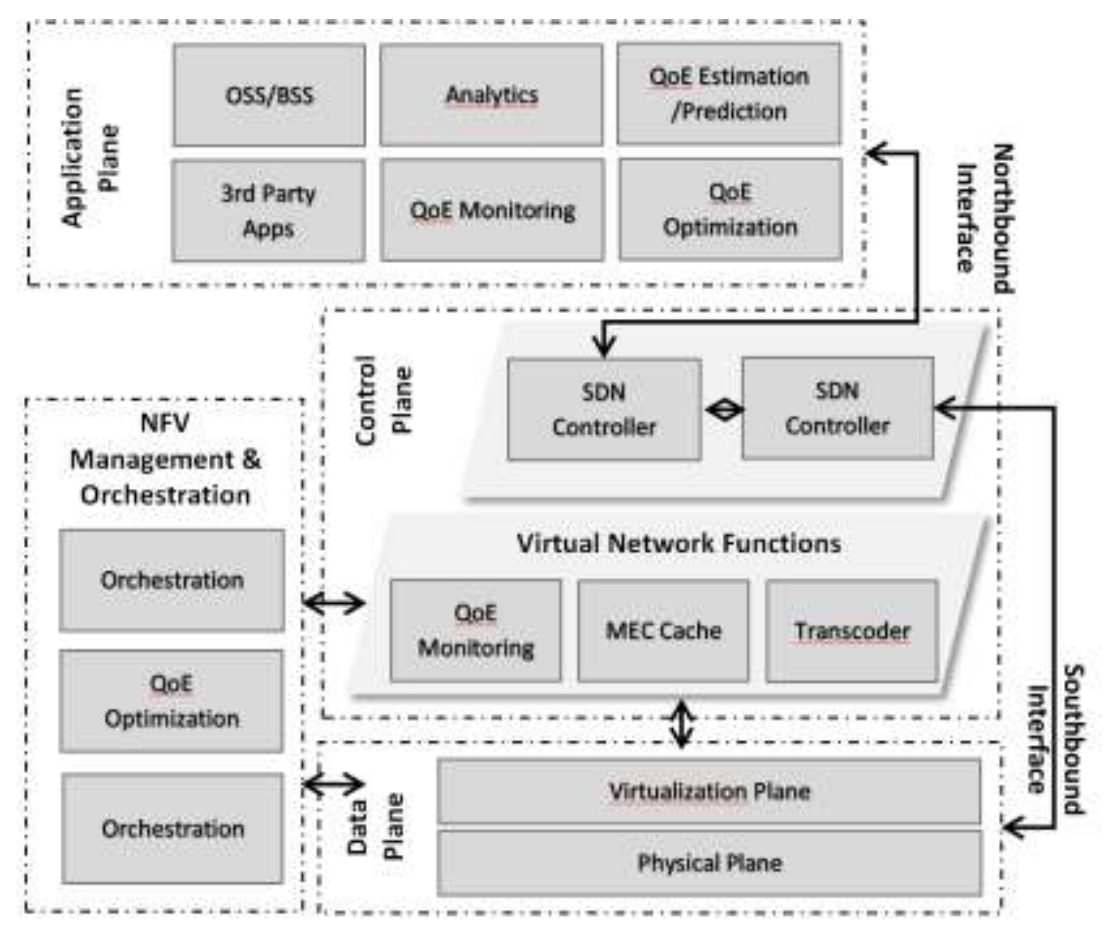

Figure 3: 5G QoE Model Analysis / Significance of QoE Model

As discussed, the SDNC's global view of the network is used to inform e.g., media elements, about how to manage quality. OpenFlow is one of the first protocol implementation to support SDN and is now widely used [31]. There are two main 
reference architectures for NFV management and orchestration (MANO), ETSI NFV MANO [32] and Open Network Automation Platform (ONAP) [33]. Figure 3 is designed with influence from MANO and has three main elements: the VNF, the NFV Infrastructure (NFVI), and NFV MANO. The main motivation behind selecting MANO is the availability of its multiple implementations [34]. To summarize, QoE-oriented VNFs co-exist with service-specific and network-specific ones, and can perform monitoring or estimation tasks based on data collected from them. That data could be fed to an optimization component within the NFV orchestrator to aid it in its tasks

In the first half of this paper we have briefly discussed the core 5G concepts and 5G-VANET requirements, communication paradigm and some relevant systems. We analyzed the importance of user experience and presented a mapping of QoE provision layers with a fundamental QoE model and $5 \mathrm{G}$ enablers.

The QoE model presented in Figure 3 is based on the core 5G concepts of SDN and NFV and decouples the control, data and application planes. As previously discussed the aim of this research is not to propose a new design and model of these concepts, however it is to use them to extrapolate quality of user experience. In our model SDNC performs resource allocation based on the global information view coupled with QoE optimization by using multiple logical entities (e.g. OpenFlow switches).

\section{CONCLUSION}

In this paper we have discussed 5G-VANETs and the research conducted in this domain. The promised ITS and respective services can achieve wise-spread acceptability and adoption only if they offer good user experience. The aim of this paper is to highlight the importance of QoE. A SDN and NVF enabled QoE model is proposed which leverages the benefits of centralized SDNC and virtualization of network hardware. Collection of QoE system influence factors and management as a VNF is achieved. This is work in progress and network level simulation is currently underway, as discussed in the following sub-section, along with some future work ideas.

\section{A. Network Simulations}

The network simulations are currently been done using Network Simulator 3 (ns3). The simulation design uses the mmWave module integrated with WAVE module. Multiple mobility models for urban and rural scenarios will be simulated and the system influence factors listed in Figure 2 will be measured.

\section{B. Subjective QoE Assessment}

The shift in performance assessment from objective Quality-of-Service (QoS) metrics to subjective end user's QoE metrics has been aided by mean opinion score (MOS), a metric rating from 1 (bad) to 5 (excellent) [2]. We will perform subjective assessment in the later part of this research.

\section{VANET as $5 G$ relay}

As part of this research, we will also explore the usage of VANET infrastructure as 5G traffic replay or forwarding. In dense urban scenario network data can be offloaded to RSU or even a vehicle and then propagated to UEs using V2V or V2P communication paradigm. It will be interesting to see the effect of such a load balancing technique on 1) service delivery and 2) network congestion.

\section{User Incentive Model}

Cost/pricing is an important aspect of overall positive user experience. In 5G-VANET, each node has resources of computing, which can be shared to maximize the utilization. The base station can offload the computing tasks to vehicles, and the vehicle can also offload the computing tasks to the BS. [Guiyang Luo1, Quan Yuan1, Haibo Zhou2]. We plan to extend the research work by analyzing a computing-node model based on some reward/incentive to the user (or even parked vehicles in this case). Such a paradigm when coupled with MEC can support novel future applications.

\section{REFERENCES}

[1] G. Karagiannis, O. Altintas, E. Ekici, G. Heijenk, B. Jarupan, K. Lin, and T. Weil, "Vehicular networking: A survey and tutorial on requirements, architectures, challenges, standards and solutions," Communications Surveys Tutorials, IEEE, vol. 13, no. 4, pp. 584-616, 2011.

[2] M. Gerla, E.K Lee, G. Pau and U. Lee, "Internet of vehicles: From intelligent grid to autonomous cars and vehicular clouds", International Journal of Distributed Sensor Networks, Hindawi Publishing Corporation, 2016, 12 (9), pp.1550147716665500

[3] Al-Fuqaha, Ala \& guizani, mohsen \& Mohammadi, Mehdi \& Aledhari, Mohammed \& Ayyash, Moussa. (2015). Internet of Things: A Survey on Enabling Technologies, Protocols and Applications. IEEE Communications Surveys \&amp Tutorials. 17. Fourthquarter 2015. 10.1109/COMST.2015.2444095.

[4] Huang, Xumin \& Yu, Rong \& Kang, Jiawen \& He, Yejun \& Zhang, Yan. (2017). Exploring Mobile Edge Computing for 5G-Enabled Software Defined Vehicular Networks. IEEE Wireless Communications. 24. 55-63. 10.1109/MWC.2017.1600387.

[5] Z. Xu, X. Li, X. Zhao, M. H. Zhang, and Z. Wang, "DSRC versus 4G-LTE for Connected Vehicle Applications: A Study on Field Experiments of Vehicular Communication Performance," Journal of Advanced Transportation, vol. 2017, Article ID 2750452, 10 pages, 2017. https://doi.org/10.1155/2017/2750452. 
[6] Q. Yuan, H. Zhou, J. Li, Z. Liu, F. Yang, and X. Shen, "Towards efficient content-delivery for automated driving services: An edge computing solution,” IEEE Network, vol. 32, no. 1, pp. 80-86, Jan./Feb. 2018

[7] G. S. Aujla, R. Chaudhary, N. Kumar, J. J. P. C. Rodrigues and A. Vinel, "Data offloading in 5g-enabled software-defined vehicular networks: A stackelberg-game-based approach", IEEE Communications Magazine 55 (8), 100-108, 2017

[8] A. Ahmed and E. Ahmed, “A survey on mobile edge computing," 10th International Conference on Intelligent Systems and Control (ISCO), pp. 18 , Jan 2016.

[9] Y. Xiao and C. Zhu, "Vehicular fog computing: Vision and challenges"” 6-9. 10.1109/PERCOMW.2017.7917508, 2017.

[10] Z. Ning, X. Wang and J. Huang, "Vehicular Fog Computing: Enabling Real-Time Traffic Management for Smart Cities". IEEE Wireless Communications. 26. 10.1109/MWC.2019.1700441, 2018.

[11] K. Zhang, Y. Mao, S. Leng, Y. He, and Y. Zhang, "Mobile-edge computing for vehicular networks: A promising network paradigm with predictive off-loading," IEEE Veh. Technol. Mag., vol. 12, no. 2, pp. 36-44, 2017.

[12] X. Hou, Y. Li, M. Chen, D. Wu, D. Jin, and S. Chen, "Vehicular fog computing: A viewpoint of vehicles as the infrastructures," IEEE Trans. Veh. Technol., vol. 65, no. 6, pp. 3860-3873, 2016.

[13] C. Campolo, A. Molinaro, A. Iera and F. Menichella, "5G Network Slicing for Vehicle-to-Everything Services", IEEE Wireless Communications, vol 24 Issue 6, pp 38-45, December 2017

[14] I. Ku, Y. Lu, M. Gerla, R. L. Gomes, F. Ongaro, and E. Cerqueira, "Towards software-defined VANET: Architecture and services,” 2014 13th Annual Mediterranean Ad Hoc Networking Workshop (MED-HOC-NET), Jun. 2014.

[15] N. Truong, G. M. Lee, and Y. Ghamri-Doudane, "Software defined networking-based vehicular Adhoc Network with Fog Computing", IEEE International Symposium on Integrated Network Management, 1202-1207. 10.1109/INM.2015.7140467, 2015.

[16] X. Wang, Z. Ning and L. Wang, "Offloading in Internet of Vehicles: A Fog-enabled Real-time Traffic Management System", IEEE Transactions on Industrial Informatics. PP. 1-1. 10.1109/TII.2018.2816590, 2018.

[17] S. Correia, A. Boukerche and R. Meneguette, "An Architecture for Hierarchical Software-Defined Vehicular Networks", IEEE Communications Magazine. 55. 80-86. 10.1109/MCOM.2017.1601105, 2017.

[18] ETSI, "Network Functions Virtualization (NFV); Architectural Frame-work,” Dec. 2014.

[19] S. Al-Sultan, M. M. Al-Doori, A. H. Al-Bayatti, and H. Zedan, "A comprehensive survey on vehicular ad hoc network," Journal of network and computer applications, vol. 37, pp. 380-392, 2014

[20] S. A. Ali, E. Ahmed, M. Imran and S. Zeadally, "5G for Vehicular Communications", IEEE Communications Magazine, DOI: 10.1109/MCOM.2018.1700467, 2018

[21] Z. Ning, X. Wang and J. Huang, "Mobile Edge Computing-Enabled 5G Vehicular Networks: Toward the Integration of Communication and Computing", IEEE Vehicular Technology Magazine. PP. 1-1. 10.1109/MVT.2018.2882873, June 2018.

[22] S. H. Bouk, S. H. Ahmed, D. Kim, and H. Song, "Named-data networking-based ITS for smart cities," IEEE Communications Magazine, vol. 55, no. 1 , pp. $105-111,2017$.

[23] M. Amadeo, C. Campolo, and A. Molinaro, "Information - centric networking for connected vehicles: a survey and future perspectives," IEEE Communications Magazine, vol. 54, no. 2, pp. 98-104, 2016.

[24] M. Garai and N. Boudriga, “A novel architecture for QoS provision on VANET”, 25-31. 10.1109/HONET.2013.6729752, 2013.

[25] International Telecommunication Union. (2017). ITU-T P.10/G.100 Vocabulary for performance, quality of service and quality of experience. [Online]. Available: http://www.itu.int.

[26] S. Baraković and L. Skorin-Kapov, "Survey and challenges of QoE management issues in wireless networks", Journal of Computer Networks and Communications, 2013

[27] L. Pierucci, "The Quality of Experience Perspective toward 5G Technology”, IEEE Wireless Communications, 2015

[28] A. Fery and S. Muhammad, "The QoE assessment model for 5G mobile technology", 1-5. 10.1109/BCWSP.2017.8272574, 2017.

[29] E. Liotou, H. Elshaer, R. Schatz, R. Irmer, M. Dohler, N. Passas and L. Merakos" Shaping QoE in the 5G ecosystem", 7th International Workshop on Quality of Multimedia Experience. 10.1109/QoMEX.2015.7148089, 2015.

[30] R. Schatz, S. Schwarzmann, T. Zinner, O. Dobr, E. Liotou, P. Počta, S. Barakovic, H. Barakovic and L. Skorin-Kapov, "QoE Management for Future Networks", 10.1007/978-3-319-90415-3_3, 2018.

[31] OpenFlow Switch Specification: Version 1.3.5 (Protocol version 0x04).

[32] ETSI, "Network Functions Virtualisation (NFV); anagement and Orchestration", 2014

[33] ONAP, “Open Network Automatoin Protocol”, https://www.onap.org/

[34] ETSI, “Open-source MANO”, https://osm.etsi.org/

[35] M. Zhu, J. Cao, Z. Cai, Z. He and M. Xu, "Providing flexible services for heterogeneous vehicles: an NFV-based approach", IEEE Network, 30(3), 64-71, 2016 